\title{
Listado de plantas ornamentales urbanas de Tunja (Boyacá, Colombia)
}

\section{A List of Urban Ornamental Plants in Tunja (Boyacá, Colombia)}

\author{
Wilson Ricardo Álvaro Alba \\ Grupo de Investigación Biología para la Conservación, Facultad de Ciencias Básicas, Escuela de Ciencias Biológicas, Universidad Pedagógica y \\ Tecnológica de Colombia \\ https://orcid.org/0000-0003-3089-9515 |walvaroa@gmail.com \\ Mónica Patricia Díaz Pita \\ Grupo de Investigación Biología para la Conservación, Facultad de Ciencias Básicas, Escuela de Ciencias Biológicas, Universidad Pedagógica y \\ Tecnológica de Colombia \\ https://orcid.org/0000-0001-9477-694X | monicadiazp@gmail.com
}

Fecha de recepción: 20 de abril de 2020

Fecha de aceptación: 29 de mayo de 2020

Sugerencia de citación: Álvaro-Alba, W. R. y Díaz-Pita, M. P. (2020). Listado de plantas ornamentales urbanas de Tunja (Boyacá, Colombia). Mutis, 10(1), 57-78. doi: 10.21789/22561498.1602

\section{RESUMEN}

Se presenta el inventario florístico de las plantas ornamentales de la ciudad de Tunja (Boyacá). Se encontraron 430 especies, distribuidas en 314 géneros y 101 familias, de las cuales 307 especies son Eudicotiledóneas, 96 Monocotiledóneas, 11 Pteridofitos, 10 Gimnospermas y 6 Magnólidas. A nivel florístico, las familias Asteraceae, Cactaceae, Orchidaceae, Solanaceae y Asparagaceae presentaron el mayor número de géneros y especies. El hábito de crecimiento más importante en términos de riqueza de especies fue el de las hierbas, seguido por los hábitos leñosos (árboles y arbustos). En ambientes de exterior se encontraron más especies de tipo arbóreo y arbustivo, mientras que en ambientes de interior predominaron las suculentas. Se registran 12 especies con algún grado de amenaza de acuerdo con la clasificación de la Unión Internacional para la Conservación de la Naturaleza (IUCN, en inglés).

Palabras clave: ambientes urbanos, diversidad de plantas vasculares, plantas de jardín, plantas de interior, riqueza florística. 


\section{ABSTRACT}

The floristic inventory of the ornamental plants of the city of Tunja (Boyacá) is presented in this paper. A total of 430 species were found, distributed in 314 genera and 101 families, of which 307 species are Eudicotyledonous, 96 Monocotyledonous, 11 Pteridophytes, 10 Gymnosperms and 6 Magnolids. At the floristic level, the families Asteraceae, Cactaceae, Orchidaceae, Solanaceae and Asparagaceae presented the highest number of genera and species. The most important growth habit in terms of species richness was that of herbs, followed by woody habits (trees and shrubs). Tree and shrub species thrive in outdoor environments, while succulents predominate indoors. Twelve species with some degree of threat, according to the International Union for Conservation of Nature (IUCN), were registered in this study.

Keywords: Floristic richness, garden plants, indoor house plants, urban environments, vascular plants diversity.

\section{INTRODUCCIÓN}

El uso de las plantas por parte del hombre se remonta a tiempos prehistóricos (Sánchez-de-Lorenzo, 2001). Las plantas ornamentales generalmente ocupaban un segundo plano de interés, al considerarse un adorno estético (Fernández, 1990), y daban cuenta de un estatus socioeconómico alto, puesto que los propietarios de los jardines eran únicamente los gobernantes, en el caso del antiguo Egipto (Fariello, 2004). La naturaleza fue la primera dueña de los jardines y es la fuente principal de ideas para su diseño. Pero es el hombre quien siempre ha ejercido una tradición cultural por la naturaleza, en especial por las flores, las cuales hacen que los actos cotidianos y simples de la humanidad estén dedicados a su utilización, significado y cuidados (Brooklyn Botanic Garden, 2001; Villegas, 1990).

Desde sus comienzos, las civilizaciones han deseado rodearse de estas plantas para admirar su belleza y así dar origen a jardines que adornan palacios, parques, edificios y mansiones privadas. Como ejemplo a destacar se encuentran los jardines colgantes de las antiquísimas ciudades de Babilonia y Nínive, que fueron considerados como una de las grandes maravillas del mundo antiguo. Desde entonces, en todas las culturas se ha dado notable importancia a la jardinería o el arte de cultivar plantas y flores con fines ornamentales (Fariello, 2004).

Colombia es considerada el país de las flores, donde el culto a ellas se destaca en todas sus manifestaciones a través de la historia, ya que ocupan un lugar muy importante en la religión, la literatura, el arte, la artesanía, los jardines de casas modernas y coloniales, las expresiones folclóricas y la ciencia. En esta última se generó un importante interés a partir de la primera Expedición Botánica, llevada a cabo en el siglo XVIII (Villegas, 1990). Las flores son importantes también para la economía del país, el cual es catalogado como el segundo exportador de este producto en el mundo (Manrique et al., 2014).

Hoy en día las plantas ornamentales no solo se emplean para adornar jardines, calles y balcones, sino que además contribuyen al mejoramiento ambiental de las ciudades, por cuanto disminuyen los niveles de dióxido de carbono atmosférico, aumentan los niveles de oxígeno mediante la fotosíntesis vegetal, modifican el microclima al proporcionar sombra y aumentar la humedad atmosférica (gracias a la transpiración foliar), son barreras protectoras contra el viento, sirven de refugio y hábitat a muchos animales, especialmente aves (Fernández, 1990), reducen el estrés laboral, favorecen la concentración y generan sensación de bienestar (Soto, 2017). Adicionalmente, los árboles representan un patrimonio histórico y emblemático de las ciudades (Mahecha et al., 2010) y junto con otros componentes arquitectónicos se utilizan para encerrar, contener, agrandar, reducir y articular el espacio exterior (Alcaldía Mayor de Bogotá, 2003). Además, amortiguan el ruido o los sonidos molestos, valorizan la propiedad, controlan la erosión y contribuyen a la estabilización de taludes (Tovar, 2007).

Por las razones antes expuestas, y debido a que actualmente se está presentando una reducción importante de las áreas silvestres a nivel mundial por la intervención del hombre, los jardines han sido catalogados como santuarios ecológicos a explorar (Brooklyn Botanic Garden, 2001). Por ello, este trabajo tiene como finalidad presentar un inventario exhaustivo de 
la flora ornamental vascular de la ciudad de Tunja, así como aportar información relacionada sobre los nombres comunes de cada especie y destacar la importancia ornamental y usos de las familias y especies botánicas más representativas de dicha ciudad.

\section{MATERIALES Y MÉTODOS}

\section{Área de estudio}

El municipio de Tunja se encuentra ubicado sobre la cordillera Oriental, en la parte central del departamento de Boyacá, localizado a $05^{\circ} 32^{\prime} 7^{\prime \prime} \mathrm{N}$ y $37^{\circ} 22^{\prime} 04^{\prime \prime} \mathrm{O}$, con alturas desde 2.700 hasta $3.150 \mathrm{~m}$, una extensión de $121,4 \mathrm{Km}^{2}$ y una temperatura de $13^{\circ} \mathrm{C}$. Limita por el norte con los municipios de Motavita y Cómbita, al oriente con los municipios de Oicatá, Chivatá, Soracá y Boyacá, por el sur con Ventaquemada y por el occidente con los municipios de Samacá, Cucaita y Sora (Alcaldía Mayor de Tunja, 2012), como se muestra en la figura 1.

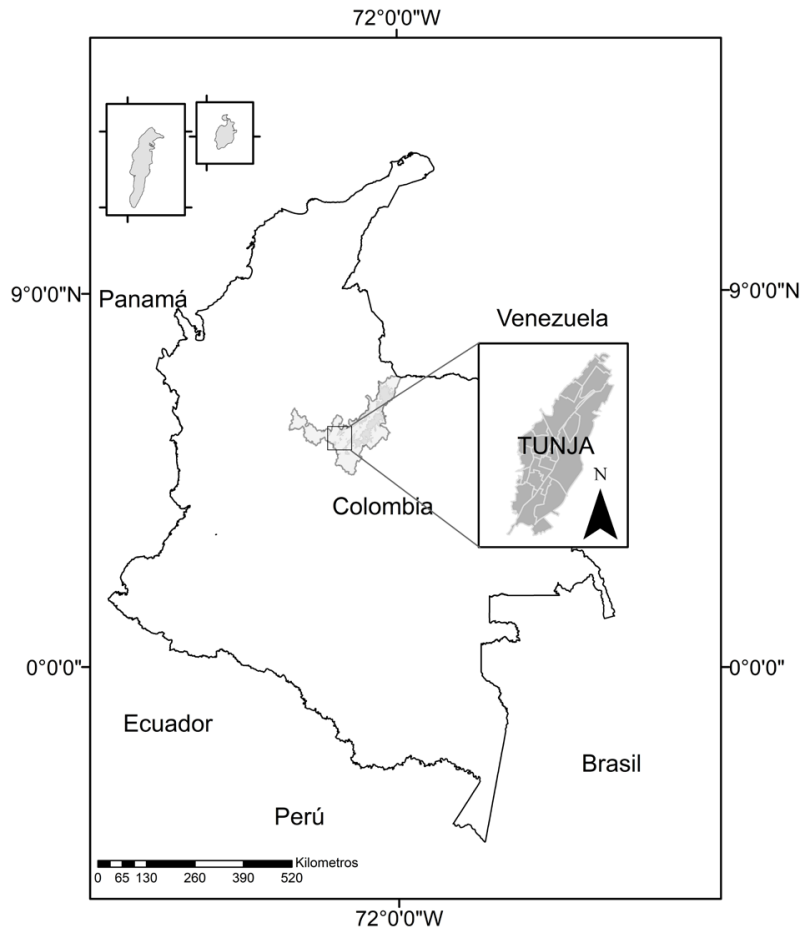

Figura 1. Ubicación del área de estudio (Tunja, Boyacá) Fuente: elaboración propia.

\section{Muestreo de plantas ornamentales}

La recopilación de información sobre las plantas ornamentales se realizó de 2016 a 2019, teniendo en cuenta las épocas secas y lluviosas. La búsqueda de las plantas se efectuó en jardines de casas y conjuntos cerrados, colegios, parques principales de la ciudad, universidades y separadores de avenidas principales. Además, se visitaron viveros e invernaderos de la ciudad de Tunja. El inventario de las especies se realizó a partir de la toma de fotografías de la planta completa y el registro de caracteres morfológicos específicos (estructuras reproductivas y presencia de exudados, entre otros) que facilitaron su posterior identificación taxonómica. Adicionalmente, se registró información relacionada con el nombre común y el ambiente en donde se encontró cada planta (interior, exterior). El hábito de crecimiento (árbol, arbusto, hierba, liana, enredadera, suculenta, sufrutescente) se determinó con base en la propuesta de Judd et al. (1999) y Mendoza y Ramírez (2006).

\section{Identificación de especies}

El material vegetal se determinó in situ y, en algunos casos, por consultas minuciosas en catálogos a partir de la revisión de las fotografías tomadas a cada una de las especies. Las claves y la literatura especializada revisada incluyen a Byrd Graf (1981), Bartholomäus et al. (1990), Molina et al. (1998), Brickell (The Royal Horticultural Society, 2000), Rodríguez (2002), Sánchez-deLorenzo (2001), Cheers (2006), Infante-Betancour et al. (2008), Sierra-Guerrero y Amarillo-Suárez (2014), Galvis (2014) y Mahecha (2015). Los sistemas de clasificación consultados siguen la propuesta de Christenhusz et al. (2011) para gimnospermas, Christenhusz et al. (2011) para pteridófitos y la de The Catalogue of Life Partnership para el caso de angiospermas (2017). La nomenclatura de las especies se corroboró en la base de datos The Plant List ${ }^{1}$ y el International Plant Name Index ${ }^{2}$ (IPNI).

\section{RESULTADOS Y DISCUSIÓN}

\section{Caracterización florística}

Para la ciudad de Tunja se registran 430 especies de plantas ornamentales (anexo 1), agrupadas en 314 géneros y 101 familias, organizadas en cinco grupos: Eudicotyledonea, Magnoliids, Monocotiledonea, Pinophyta y Pteridófitos (tabla 1).

1 http://www.theplantlist.org

2 https://www.ipni.org 
Tabla 1. Inventario florístico de plantas ornamentales de Tunja

\begin{tabular}{|c|c|c|c|}
\hline Clase & $\begin{array}{c}\text { N.ㅇ } \\
\text { familias }\end{array}$ & $\begin{array}{c}\text { N.․ } \\
\text { géneros }\end{array}$ & $\begin{array}{c}\text { N.․ } \\
\text { especies }\end{array}$ \\
\hline Eudicotyledoneae & 72 & 222 & 307 \\
\hline Magnoliids & 2 & 3 & 6 \\
\hline Monocotiledónea & 16 & 73 & 96 \\
\hline Pinophyta & 5 & 7 & 10 \\
\hline Pteridophyta & 6 & 9 & 11 \\
\hline Total & 101 & 314 & 430 \\
\hline
\end{tabular}

Fuente: elaboración propia.

Las familias con el mayor número de géneros y especies son Asteraceae (30 géneros, 36 especies), Cactaceae $(16,20)$, Orchidaceae $(13,15)$, Solanaceae $(13$, $15)$, Asparagaceae $(11,18)$, Araceae $(9,14)$ y Crassulaceae $(7,19)$, como se detalla en la tabla 2 . El $80 \%$ de las familias (81) tienen menos de cinco géneros. Así mismo, $78 \%$ de las familias (79) se encuentran representadas por menos de seis especies.

Tabla 2. Familias con mayor riqueza de géneros y especies de plantas ornamentales en Tunja

\begin{tabular}{ccc}
\hline Familias & $\begin{array}{c}\text { N.o géneros y } \\
\text { porcentaje (\%) }\end{array}$ & $\begin{array}{c}\text { N.o especies y } \\
\text { porcentaje (\%) }\end{array}$ \\
\hline Asteraceae & $30(9,6)$ & $36(8,4)$ \\
\hline Cactaceae & $16(5,1)$ & $20(4,7)$ \\
\hline Orchidaceae & $13(4,1)$ & $15(3,5)$ \\
\hline Solanaceae & $13(4,1)$ & $15(3,5)$ \\
\hline Asparagaceae & $11(3,5)$ & $18(4,2)$ \\
\hline Araceae & $9(2,9)$ & $14(3,3)$ \\
\hline Crassulaceae & $7(2,2)$ & $19(4,4)$ \\
\hline Fabaceae & $7(2,2)$ & $11(2,6)$ \\
\hline Acanthaceae & $7(2,2)$ & $11(2,6)$ \\
\hline Lamiaceae & $7(2,2)$ & $10(2,3)$ \\
\hline Malvaceae & $6(1,9)$ & $8(1,9)$ \\
\hline Asphodelaceae & $6(1,9)$ & $7(1,6)$ \\
\hline Euphorbiaceae & $5(1,6)$ & $11(2,6)$ \\
\hline
\end{tabular}

Fuente: elaboración propia.
Las Asteráceas son ampliamente cultivadas en parques y jardines por el atractivo de sus inflorescencias, follaje o fructificación (Del-Vitto \& Petenatti, 2015). Algunas especies son muy atractivas por sus llamativos capítulos de flores liguladas, como es el caso de Achillea millefolium L., Argyranthemum frutescens (L.) Sch. Bip., Aster amellus L., Calendula officinalis L., CaIlistephus chinensis (L.) Nees, Chamaemelum nobile (L.) All., Chrysanthemum morifolium Ramat., Coreopsis auriculata L., Dahlia spp., Erigeron karvinskianus DC., Felicia drakensbergensis J.M. Wood \& M.S. Evans, Gaillardia x grandiflora Van Houtte, Gazania rigens (L.) Gaertn., Ismelia carinata (Schousb.) Sch.Bip., Helichrysum bracteatum (Vent.) Haw., Leucanthemum vulgare Lam., Osteospermum spp., Pericallis hybrida B. Nord., Tagetes spp. y Zinnia peruviana (L.) L.; las que tienen capítulos de flores flosculosas vistosas, como Silybum marianum (L.) Gaertn.; aquellas utilizadas como flor de corte, Gerbera jamesonii Adlam, Helianthus annuus L.; otras cuyas inflorescencias resultan poco vistosas pero que compensan este rasgo con un follaje blanco-lanoso o discolor, Centaurea cineraria L., Helichrysum petiolare Hilliard \& B.L. Burtt, Leucophyta brownii Cass., Santolina chamaecyparissus L., Jacobaea maritima (L.) Pelser \& Meijden, Senecio niveoaureus Cuatrec.; las enredaderas, Senecio tamoides DC.; las de estrato arbóreo, como Smallanthus pyramidalis (Triana) H. Rob.; las suculentas, Senecio rowleyanus $\mathrm{H}$. Jacobsen.; y las de uso potencial para reforestación, como Baccharis latifolia (Ruiz \& Pav.) Pers.

Las cactáceas son muy abundantes en los viveros y tienen una alta demanda por ventajas relacionadas con el cuidado mínimo que requieren. Según Bueno y Plumed (2017), estas plantas necesitan regarse con poca frecuencia, toleran altas temperaturas y crecen en una amplia variedad de sustratos. Además, especies del género Mammillaria son utilizadas para capturar las radiaciones emitidas por los monitores de computadores (Grijalva, 2006). El $47 \%$ de los cactus está en peligro y el $86 \%$ de los que se utilizan en horticultura provienen de poblaciones silvestres en peligro de extinción - más que de su cultivo- debido a que la reproducción de diversas especies tarda mucho tiempo (Bizberg, 2016), como es el caso de algunas especies de los géneros Melocactus y Pilosocereus. Algunas especies de cactus se pueden utilizar como cercas vivas; por ejemplo, Acanthocereus tetragonus (L.) Hummelinck, Austrocylindropuntia cylindrica (Lam.) Backeb., 
Austrocylindropuntia subulata (Muehlenpf.) Backeb., Cereus hexagonus (L.) Mill., Opuntia caracassana Salm-Dyck, Opuntia microdasys (Lehm.) Pfeiff., Opuntia microdasys subsp. rufida (Engelm.) U. Guzmán \& Mandujano, Pilosocereus lanuginosus (L.) Byles \& G.D.Rowley y Stenocereus griseus (Haw.) Buxb.

Colombia es el país con mayor diversidad de especies de orquídeas, las cuales se destacan como ornamentales por lo vistoso de sus flores y porque presentan diversos tamaños, colores y variedad de formas, como en los géneros, Anguloa, Arundina, Cattleya, Epidendrum, Masdevallia, Miltoniopsis, Paphiopedilum, Phalaenopsis, Phragmipedium, entre otros. Según Castellanos y Torres (2018), desde hace varios años existe la cultura de recolectar plantas silvestres por parte de expertos y aficionados, quienes las venden a coleccionistas y comerciantes. Actualmente, existen varios híbridos con valor ornamental obtenidos a partir de técnicas de propagación in vitro, lo cual ha permitido que las personas puedan adquirir una gran diversidad de especies de forma más accesible.

Nuestros hallazgos permiten concluir que los géneros con mayor riqueza de especies son Begonia, Euphorbia, Ficus, Fuchsia y Oxalis (tabla 3) y que la mayoría de los géneros (96\%) incluyen de una a tres especies.

Tabla 3. Géneros con mayor número de especies y porcentaje con relación al total de especies ornamentales de Tunja.

\begin{tabular}{cc}
\hline Géneros & N.o de especies y porcentaje (\%) \\
\hline Begonia & $9(2,1)$ \\
\hline Euphorbia & $6(1,4)$ \\
\hline Ficus & $6(1,4)$ \\
\hline Fuchsia & $5(1,2)$ \\
\hline Oxalis & $5(1,2)$ \\
\hline Acacia & $4(0,9)$ \\
\hline Bryophyllum & $4(0,9)$ \\
\hline Dianthus & $4(0,9)$ \\
\hline Opuntia & $4(0,9)$ \\
\hline Pelargonium & $4(0,9)$ \\
\hline Peperomia & $4(0,9)$ \\
\hline Sedum & $4(0,9)$ \\
\hline
\end{tabular}

Fuente: elaboración propia.
El género Begonia se distingue por su importancia económica y valor ornamental. El cultivo de este género es de gran interés por la forma y el color vistoso de sus hojas, así como por su fácil propagación y cuidado (Jiménez \& Schubert, 1997). Las especies de este género se encontraron principalmente al interior de casas y unas pocas especies, como Begonia cucullata var. hookeri L.B. Sm. \& B.G. Schub. y Begonia maculata Raddi, se observaron en jardines de universidades, colegios, empresas y parques.

Las especies arbustivas del género Euphorbia se cultivan por sus hojas o las brácteas coloreadas que rodean a las flores (Sánchez-de-Lorenzo, 2001), como es el caso de Euphorbia pulcherrima Willd. ex Klotzsch, Euphorbia milii Des Moul. y Euphorbia cotinifolia L. Otras especies tienen aspecto cactiforme, carecen de hojas (o están reducidas) y, en ocasiones, están provistas de espinas. Las especies de hábito suculento presentan tallos adaptados para el almacenamiento de agua y cumplen las funciones de asimilación que corresponden a las hojas (Sánchez-de-Lorenzo, 2001), como Euphorbia tirucalli L., Euphorbia trigona Mill. y Euphorbia tithymaloides L.

En general, las especies de Ficus han sido utilizadas para proporcionar sombra y protección de cuencas, gracias a sus copas densas, evitar la erosión de terrenos, al poseer una gran formación de raíces, y también como cercas vivas, alimento para aves y ornamentales (Ibarra-Manríquez et al. 2012). El cultivo de especies arbóreas atrae una importante diversidad de aves por sus frutos carnosos, lo cual facilita la dispersión de semillas y favorece su crecimiento, puesto que estas especies presentan gran adaptabilidad a diferentes condiciones ambientales (Valverde \& Hine, 2002), como es el caso de Ficus soatensis Dugand, Ficus tequendamae Dugand y Ficus velutina Willd., las cuales se encuentran en separadores viales, senderos peatonales, campus universitarios y andenes. También se destacan Ficus benjamina L., especie cultivada al interior de casas y edificios y en algunos espacios exteriores, donde es empleada como cerca viva en parques y jardines, y Ficus pumila L., arbusto de hábito trepador que cubre paredes de casas modernas. 


\section{Hábito de crecimiento}

Las familias botánicas que forman las plantas crasas son Cactaceae, Crassulaceae, Asphodelaceae, Aizoaceae y Euphorbiaceae, aunque otras familias como Apocynaceae y Asparagaceae también tienen representantes de plantas suculentas. De acuerdo con Sánchez-de-Lorenzo (2001), las crasas son útiles y combinan bien con rocas y gravas volcánicas de diversas tonalidades, además responden muy bien a la multiplicación vegetativa y en general son de fácil cultivo; en particular algunas suculentas.

El hábito predominante en términos de riqueza fue el de las hierbas $(45,1 \%)$, seguido por los hábitos arbustos, suculentas y árboles. Los demás hábitos de crecimiento presentaron menos de 20 especies (tabla 4). Las hierbas están mejor representadas por las familias Asteraceae (23 especies), Orchidaceae (15) y Araceae (13). En árboles, la familia dominante en número de especies es Fabaceae (10), seguida de Moraceae (5); las demás familias presentaron de 1 a 3 especies. Las familias de arbustos más representativas son Malvaceae y Solanaceae (7 especies cada una), Euphorbiaceae (6) y Onagraceae (5). En cuanto a las suculentas, la familia Cactaceae fue el grupo mejor representado, con 20 especies, seguida de Crassulaceae, con 19. Para el hábito sufrutescente, la familia Asteraceae (6) presenta el mayor número de especies. Las familias más importantes dentro de las lianas y enredaderas son Apocynaceae y Bignoniaceae (tres especies cada una) y Convolvulaceae y Lamiaceae (dos especies cada una).

Las plantas ornamentales herbáceas se encontraron en todos los ambientes, ya que son fácilmente cultivadas por la belleza de su follaje y sus flores, su fácil reproducción o propagación de las especies y por su tamaño, debido a que no requieren espacios muy amplios y son ideales en jardines e interiores de casas. Los árboles y arbustos están restringidos a parques, separadores de vías y campus universitarios, donde los espacios son más abiertos.

\section{Tipo de ambiente}

La mayor riqueza de especies de plantas ornamentales registradas para cada ambiente en Tunja se encuentran creciendo en el exterior (251); en menor proporción se presentan las plantas de interior (144). Adicionalmente, en la ciudad se encuentran especies
Tabla 4. Número de especies de plantas ornamentales por hábitos de crecimiento

\begin{tabular}{|c|c|c|}
\hline Hábito & N.o de especies & Porcentaje (\%) \\
\hline Árbol & 56 & 13 \\
\hline Arbusto & 79 & 18,4 \\
\hline Enredadera & 8 & 1,9 \\
\hline Hierba & 194 & 45,1 \\
\hline Liana & 17 & 3,7 \\
\hline Suculenta & 58 & 13,5 \\
\hline Sufrutescente & 18 & 4,2 \\
\hline
\end{tabular}

Fuente: elaboración propia.

que pueden crecer tanto en interior como en exterior, las cuales se denominan mixtas (35), como se muestra en la tabla 5. Los géneros más representativos para ambientes de exterior son Ficus, Fuchsia, Acacia, Dianthus y Pelargonium. En ambientes de interior se destacan Begonia, Euphorbia, Bryophyllum y Sedum. Los géneros Oxalis, Citrus y Tradeschantia son los más comunes en ambientes mixtos.

En el exterior, los hábitos más representativos son hierbas, arbustos y árboles. En ambientes de interior, los más importantes son las hierbas y las suculentas. Por su parte, en los ambientes mixtos dominaron las especies de hábito herbáceo (tabla 5). En los ambientes de exterior, para hierbas, las familias más representativas son Asteraceae (21 especies) y Iridaceae (6), seguidas de Amaryllidaceae, Caryophyllaceae, Geraniaceae, Plantaginaceae, Poaceae y Solanaceae, con cuatro especies cada una. En hábitos leñosos, las familias más importantes en términos de riqueza son Fabaceae (10 especies), Solanaceae, Myrtaceae y Malvaceae (7 especies cada una), Onagraceae y Moraceae (5 cada una) y Asteraceae (4 especies).

En ambientes de interior, las familias de herbáceas más importantes son Orchidaceae (14 especies), Araceae, Begoniaceae y Bromeliaceae (7 cada una) y Maranthaceae (5). Dentro de suculentas, destacan las familias Cactaceae (18 especies), Crassulaceae (14) y Euphorbiaceae (3). Respecto a las especies que crecen en ambos ambientes (mixtas), las familias de herbáceas más representativas son Asparagaceae, Commelinaceae y Oxalidaceae con tres especies cada una. 
Tabla 5. Riqueza de especies ornamentales en cada ambiente para los hábitos de crecimiento registrados en el área de estudio

\begin{tabular}{ccccc}
\hline & \multicolumn{4}{c}{ N.o de especies por ambiente } \\
\cline { 2 - 5 } Hábito & Exterior & Interior & Mixta & Total \\
\hline Árbol & 51 & - & 5 & 56 \\
\hline Arbusto & 66 & 9 & 4 & 79 \\
\hline Enredadera & 6 & 2 & - & 8 \\
\hline Hierba & 88 & 82 & 24 & 194 \\
\hline Liana & 15 & 2 & - & 17 \\
\hline Suculenta & 14 & 42 & 2 & 58 \\
\hline Sufrutescente & 11 & 7 & - & 18 \\
\hline Total & 251 & 144 & 35 & 430 \\
\hline
\end{tabular}

Fuente: elaboración propia.

De las 430 especies ornamentales registradas para Tunja, 12 presentan algún grado de amenaza, de acuerdo con datos de la Unión Internacional para la Conservación de la Naturaleza ${ }^{3}$ (IUCN, en inglés); Brugmansia arborea (L.) Lagerh. y Brugmansia sanguinea (Ruiz \& Pav.) D.Don son consideradas como extintas en estado silvestre (EW); tres especies están en la categoría en peligro [EN] (Bryophyllum daigremontianum (Raym.-Hamet \& H.Perrier) A. Berger, Pinus radiata D. Don, Schefflera elegantissima (Veitch ex Mast.) Lowry \& Frodin); y 7 están clasificadas como vulnerables [vu] (Armeria maritima [Mill.] WiIld., Araucaria heterophylla [Salisb.] Franco, Dionaea muscipula J. Ellis, Hatiora gaertneri [Regel] Barthlo$\mathrm{tt}$, Limonium perezii [Stapf] F.T. Hubb., Schlumbergera truncata [Haw.] Moran, Retrophyllum rospigliosii [Pilg.] C.N. Page). De acuerdo con lo observado en la fase de campo, estas especies no son muy comunes; excepto $P$. radiata y $R$. rospigliosii, que se encuentran en parques, campus universitarios y separadores viales de la ciudad.

La ciudad de Tunja cuenta con 114 especies de origen neotropical que se emplean como ornamentales. De acuerdo con la Resolución 1912 del Ministerio de Ambiente y Desarrollo Sostenible (2018), del total de estas especies, seis se encuentran con algún grado de

www.iucnredlist.org amenaza en el país: Ceroxylon quindiuense (H.Karst.) H.Wendl. (Arecaceae), Juglans neotropica Diels (Juglandaceae), Anguloa clowesii Lindl. (Orchidaceae) y Cattleya trianae Linden \& Rchb. f. (Orchidaceae), categorizadas como en peligro (EN), y Quercus humboldtii Bonpl. (Fagaceae), y Miltoniopsis vexillaria (Rchb. f.) God.-Leb. (Orchidaceae), consideradas vulnerables (vu). Las hierbas están representadas con 42,1\% del total de hábitos de crecimiento, los árboles y arbustos con $36,8 \%$ y las suculentas con $13,2 \%$. Los demás hábitos de crecimiento registran entre 1,7 y 3,5\%. La importancia del cultivo de especies nativas en la ciudad podría favorecer un aumento en la fauna nativa asociada (Sierra \& Amarillo, 2014), como es el caso de las Solanáceas, Brugmansia sanguinea (Ruiz \& Pav.) D. Don, y Streptosolen jamesonii (Benth.) Miers, las cuales son polinizadas por colibríes.

\section{CONCLUSIONES}

Es recomendable la preferencia por las plantas nativas de uso ornamental para adornar avenidas, parques, campus universitarios y calles de la ciudad, proporcionando a la población espacios sombreados que generen microclimas agradables, contribuyan al mejoramiento de la calidad de vida de las personas, minimicen los efectos de la actividad antrópica y aporten a la conservación de la diversidad local. Lo anterior, teniendo en cuenta que la vegetación nativa tiene fauna asociada a la cual le proporciona alimento y hábitat, y esta, a su vez, realiza la dispersión de semillas y polinización, favoreciendo la ampliación de la cobertura vegetal.

Dentro de las especies de origen neotropical que pueden tener utilidad como cerca viva se encuentran: Lantana camara L. (sanguinaria), Streptosolen jamesonii (Benth.) Miers (mermelada) y algunas especies de la familia Cactaceae, como plantas trepadoras para muros, Solanum laxum Spreng. (manto de maría), y Solandra maxima (Moc. \& Sessé ex Dunal) P.S.Green (copa de oro). Para separadores viales amplios, campus universitarios y parques destacan: Schinus moIle L. (muelle), Yucca gigantea Lem. (yuca), Baccharis latifolia (Ruiz \& Pav.) Pers. (chilco), Vallea stipularis L.f. (campano, sanjuanito, raque), Escallonia pendula (Ruiz \& Pav.) Pers. (mangle), Croton coriaceus Kunth (sangregrado, drago, croto), Caesalpinia spinosa (Molina) Kuntze (dividivi), Erythrina rubrinervia Kunth (chocho), Senna multiglandulosa (Jacq.) H.S. Irwin \& 
Barneby (alcaparro enano), Senna viarum (Little) H.S. Irwin \& Barneby (alcaparro gigante), Quercus humboldtii Bonpl. (roble), Juglans neotropica Diels (cedro nogal), Lafoensia acuminata (Ruiz \& Pav.) DC. (guayacán de Manizales), Tibouchina lepidota (Bonpl.) Baill. (siete cueros), Cedrela montana Moritz ex Turcz. (cedro), Ficus spp., Ledenbergia seguierioides Klotzsch ex Moq. (mil flores), Retrophyllum rospigliosii (Pilg.) C.N. Page (pino colombiano), Abatia parviflora Ruiz \& Pav. (duraznillo, velitas), Salix humboldtiana Willd. (sáuce). Para senderos se encuentran: Canna indica L. (achira) Cuphea ignea A. DC. (cigarrillitos), Begonia cucullata var. hookeri (A.DC.) L.B.Sm. \& B.G.Schub. (pichones), Duranta erecta L. (duranta). Para jardines verticales: Fittonia albivenis (Lindl. ex Veitch) Brummitt (abre caminos, fitonia) y Phlebodium aureum (L.) J. Sm. (helecho blue star). Por último, como tapizantes sobresalen Erigeron karvinskianus DC. (margarita, manzaniIla), Centradenia grandifolia (Schltdl.) Endl. (siete cueros rastrero) y Calibrachoa sp. (Calibranchoa).

\section{AGRADECIMIENTOS}

A José Manuel Sánchez de Lorenzo por la determinación de algunas plantas. A Daniela Porras y Juliana Montes por la identificación de algunas especies de cactus. A Wilber Ruíz por los aportes y las sugerencias en la toma de fotografías necesarias para la identificación de las especies. A Celeste Montilla por la elaboración del mapa. A Yeimy Olarte por su colaboración en la confirmación de los nombres comunes de algunas plantas. A los viveros y a la comunidad de Tunja por su valioso tiempo, colaboración y cordial atención.

\section{REFERENCIAS}

Alcaldía Mayor de Bogotá (2003). Beneficios de los árboles en el ambiente urbano. http://www. ambientebogota.gov.co/c/document_library/ get_file?uuid=1ff93b18-ecc1-4d06-a83c820920dd0408\&groupld=10157

Alcaldía Mayor de Tunja (2012). Plan de desarrollo municipal 2012-2015. Hechos de verdad. por una Tunja más humana, saludable, segura y sostenible. Alcaldía Mayor de Tunja.

Bartholomäus, A., De-la-Rosa, A., Santos, J., Acero, L., \& Moosbrugger. W. (1990). El Manto de la Tierra.
Flora de los Andes. Guía de 150 especies de la flora andina. Lerner.

Bizberg, A. (2016, marzo 30). Tierra de cactus. Cienciorama. http://www.cienciorama.unam. $\mathrm{mx} / \# !$ titulo/460/?tierra-de-cactus

Brooklyn Botanic Garden (2001). El jardinero ambiental. Brooklyn Botanic Garden.

Bueno, V., \& Plumed, J. (2017). Monografías botánicas. Los Cactus. Universitat de València.

Castellanos, C., \& Torres, G. (2018). Orquídeas de Cundinamarca: conservación y aprovechamiento sostenible. Instituto de Investigación de Recursos Biológicos Alexander von Humboldt, Pontificia Universidad Javeriana, Jardín Botánico de Bogotá "José Celestino Mutis", Corporación Colombiana de Investigación Agropecuaria (Corpoica) \& Gobernación de Cundinamarca.

Cheers, G. (ed.). (2006). Botánica. Guía ilustrada de plantas más de 10.000 especies de la A la Z y cómo cultivarlas. Könemann.

Christenhusz, M. J., Reveal, J. L., Farjon, A., Gardner, M. F., Mill, R. R., \& Chase, M. W. (2011). A new classification and linear sequence of extant gymnosperms. Phytotaxa, 19, 55-70. http://dx.doi. org/10.11646/phytotaxa.19.1.3

Christenhusz, M. J., Zhang, X., \& Schneider, H. (2011). A linear sequence of extant families and genera of lycophytes and ferns. Phytotaxa, 19, 7-54. http:// dx.doi.org/10.11646/phytotaxa.19.1.2

Del-Vitto, L. A., \& Petenatti, E. M. (2015). Asteráceas de importancia económica y ambiental Segunda parte: Otras plantas útiles y nocivas. Multequina, 24, 47-74.

Fariello, F. (2004). La Arquitectura de los jardines: de la antigüedad al siglo xx (segunda edición). Reverté S.A.

Fernández, E. (1990). Las plantas ornamentales oriundas de América. En J. Fernández \& I. González, La agricultura viajera. Cultivos y manufacturas de plantas industriales y alimentarias en España $y$ en la América Virreinal. Consejo Superior de Investigación. Ministerio de Agricultura, Pesca y Alimentación. 
Galvis, H. (2014). Colores para el jardín tropical: guía de plantas ornamentales (primera edición). Grijalbo.

Graf, B. A. (1981). Tropica: Color cyclopedia of exotic plants and trees (segunda edición). Roehrs Company East Rutherford.

Grijalva, A. (2006). Flora útil etnobotánica de Nicaragua (primera edición). Ministerio del Ambiente y los Recursos Naturales de Nicaragua.

Ibarra-Manríquez, G., Cornejo-Tenorio, G., GonzálezCastañeda, N., Piedra-Malagón, E. M., \& Luna, A. (2012). El género Ficus L. (Moraceae) en México. Botanical Sciences, 90(4), 389-452. https://doi. org/10.17129/botsci.472

Infante-Betancour J., Jara-Muñoz A., \& Rivera-Díaz, O. (2008). Árboles y arbustos más frecuentes de la Universidad Nacional de Colombia, sede Bogotá. Universidad Nacional de Colombia.

International Plant Names Index [IPNI]. (2020). International Plant Names Index. The Royal Botanic Gardens, Kew, The Harvard University Herbaria \&The Australian National Herbarium.

International Union for Conservation of Nature [IUCN]. 2020. The IUCN Red List of Threatened Species. IUCN. https://www.iucnredlist.org

Jiménez, R., \& Schubert, B. G. (1997). Begoniaceae. Flora de Veracruz, 100, 1-70.

Judd, W. S., Campbell, C. S., Kellogg, E. A., Stevens, P. F., \& Donoghue, M. J. (1999). Plant systematics: A phylogenetic approach. Sinauer Associates Inc.

Mahecha, G., Sánchez, F., Chaparro, J., Cadena, H., Tovar, G., Villota, L., Morales, G., Castro, J. A., Bocanegra, F., \& Quintero, M. (2010). Arbolado urbano de Bogotá. Identificación, descripción y bases para su manejo. Alcaldía Mayor de Bogotá.

Mahecha, G. (2015). Jardinería urbana de Bogotá: guía ilustrada. Jardín Botánico de Bogotá "José Celestino Mutis".

Manrique, L. N., Zuleta, D., Agudelo, A. F., Burgos, S. A., Jerez, D., Mejía, J. D., Pereira, M. P., \& Palacio, V. (2014). Floricultura colombiana en contexto: experiencias y oportunidades en Asia pacífico. Online Journal Mundo Asia Pacífico, 3(5), 52-79. https://doi.org/10.17230/map.v3.i5.03

Mendoza, H., \& Ramírez, B. (2006). Guía ilustrada de géneros de Melastomataceae y Memecylaceae de Colombia. Instituto de Investigación de Recursos Biológicos Alexander von Humboldt \& Universidad del Cauca.

Ministerio de Ambiente y Desarrollo Sostenible de Colombia (2018). Lista de especies silvestres amenazadas de la diversidad biológica continental y marino-costera de Colombia. Resolución 1912 de 2017 expedida por el Ministerio de Ambiente y Desarrollo Sostenible.v2.3. Ministerio de Ambiente y Desarrollo Sostenible de Colombia. https://ipt. biodiversidad.co/sib/resource? $r=$ resolucion19122017mads

Molina, L. F., Uribe, E., \& Osorio, J. (1998). Las Flores de los jardines de Santa Fe de Bogotá. Alcaldía Mayor de Bogotá.

Rodríguez, W. (2002). Helechos, licopodios, selaginelas $y$ equisetos del Parque Regional Arví (primera edición). CORANTIOQUIA \& Jardín Botánico Joaquín Antonio Uribe.

Sánchez-de-Lorenzo, J. M. (2001). Guía de las plantas ornamentales. Mundi-Prensa.

Sierra-Guerrero, M. C., \& Amarillo-Suárez, A. R. (2014). Catálogo de la vegetación en jardines domésticos de Bogotá, Colombia. Biota Colombiana, 15(1), 1046

Soto, A. (2017). Guía básica de plantas ornamentales para el diseño de jardines de interior y exterior para clima templado [tesis de pregrado, Universidad Rafael Landívar]. Repositorio Universidad Rafael Landívar.

The Catalogue of Life Partnership (2017). APG IV: Angiosperm Phylogeny Group classification for the orders and families of flowering plants [Data set]. The Catalogue of Life Partnership. https://www. gbif.org/dataset/fa8ab13c-52ed-4754-b838aeff74c79718 
The Royal Horticultural Society (2000). Nueva enciclopedia de plantas y flores (tercera edición). Grijalbo.

Tovar, G. (2007). Manejo del arbolado urbano en Bogotá. Territorios, 16-17, 149-173.

Valverde, L., \& Hine, A. (2002). Germinación y micropropagación de Ficus obtusifolia, F. jimenezii y F. morazaniana (Moraceae). Uniciencia, 19(1), 77-82.

Villegas, B. (1990). Colombia, tierra de flores. Villegas Editores. 
Anexo 1. Listado de especies por familia, nombre científico, nombre común y hábito de crecimiento.

(Va desde pág. 68 a pág. 79).

*Especies neotropicales.

**Ab: árbol; Ar: arbusto; Hb: hierba; En: enredadera; Ln: liana; Sc: suculenta; Sf: sufrutescente.

\begin{tabular}{|c|c|c|c|}
\hline Familia & Nombre científico & Nombre común & Hábito** \\
\hline ACANTHACEAE & Acanthus mollis L. & Acanto & $\mathrm{Hb}$ \\
\hline ACANTHACEAE & Aphelandra arborea (Mill.) M.R.Almeida * & Afelandra & Sf \\
\hline ACANTHACEAE & Aphelandra squarrosa Nees & Cebra, afelandra & Sf \\
\hline ACANTHACEAE & Fittonia albivenis (Lindl. ex Veitch) Brummitt * & Abre caminos, fitonia & $\mathrm{Hb}$ \\
\hline ACANTHACEAE & Hypoestes phyllostachya Baker & Polka & $\mathrm{Hb}$ \\
\hline ACANTHACEAE & Justicia brandegeeana Wassh. \& L.B.Sm. & Camarón rojo & Sf \\
\hline ACANTHACEAE & Justicia carnea Lindl. * & Jacobinia, jacobina & $\mathrm{Ab}$ \\
\hline ACANTHACEAE & Pachystachys lutea Nees* & Camarón amarillo & $\mathrm{Ab}$ \\
\hline ACANTHACEAE & Thunbergia alata Bojer ex Sims & Ojo de poeta, susanitas & En \\
\hline ACANTHACEAE & Thunbergia grandiflora (Roxb. ex Rottl.) Roxb. & Quinceañera & Ln \\
\hline ACANTHACEAE & Thunbergia mysorensis (Wight) T.Anderson & Cortina china & Ln \\
\hline ADOXACEAE & Sambucus nigra L. & Saúco & $\mathrm{Ab}$ \\
\hline AIZOACEAE & Lampranthus deltoides (L.) Glen ex Wijnands & Granizo & Sf \\
\hline AIZOACEAE & Lampranthus spectabilis (Haw.) N.E. Br. & Brilla a las once & Sf \\
\hline AIZOACEAE & Mesembryanthemum cordifolium L.f. & Suculenta & Sc \\
\hline AIZOACEAE & Pleiospilos nelii Schwantes & Cactus de piedra & Sc \\
\hline ALSTROEMERIACEAE & Alstroemeria sp. * & Astromelia & $\mathrm{Hb}$ \\
\hline ALTINGIACEAE & Liquidambar formosana Hance & Ámbar & $\operatorname{Ar}$ \\
\hline AMARANTHACEAE & Celosia argentea $\mathrm{L}$. & Cresta de gallo, plumón & $\mathrm{Hb}$ \\
\hline AMARANTHACEAE & Gomphrena globosa L. & Gonfrena & $\mathrm{Hb}$ \\
\hline AMARANTHACEAE & Iresine diffusa fo. herbstii (Hook.) Pedersen & Amaranto & $\mathrm{Hb}$ \\
\hline AMARYLLIDACEAE & Agapanthus praecox subsp. orientalis F.M.Leight. & Agapanto & $\mathrm{Hb}$ \\
\hline AMARYLLIDACEAE & Caliphruria subedentata Baker * & Billete o billetón & $\mathrm{Hb}$ \\
\hline AMARYLLIDACEAE & Clivia miniata (Lindl.) Bosse & Clivia & $\mathrm{Hb}$ \\
\hline AMARYLLIDACEAE & Crinum x powellii Baker & Azucena blanca & $\mathrm{Hb}$ \\
\hline AMARYLLIDACEAE & Hippeastrum vittatum (L’Hér.) Herb. & Azucena roja & $\mathrm{Hb}$ \\
\hline AMARYLLIDACEAE & Zephyranthes rosea Lindl. * & Lirio de lluvia & $\mathrm{Hb}$ \\
\hline ANACAMPSEROTACEAE & Anacampseros telephiastrum DC. & Suculenta & $\mathrm{Hb}$ \\
\hline ANACARDIACEAE & Schinus molle L. * & Muelle, falso pimiento & $\mathrm{Ar}$ \\
\hline APOCYNACEAE & Adenium obesum (Forssk.) Roem. \& Schult. & Flor del desierto & $\mathrm{Sc}$ \\
\hline APOCYNACEAE & Hoya carnosa (L.f.) R.Br. & Hoya & Ln \\
\hline APOCYNACEAE & Mandevilla boliviensis (Hook.f.) Woodson * & Mandevila & Ln \\
\hline APOCYNACEAE & Mandevilla sanderi (Hemsl.) Woodson & Diplodemia & Ln \\
\hline
\end{tabular}




\begin{tabular}{|c|c|c|c|}
\hline Familia & Nombre científico & Nombre común & Hábito** \\
\hline APOCYNACEAE & Nerium oleander L. & Adelfa & $\mathrm{Ab}$ \\
\hline APOCYNACEAE & Vinca major $\mathrm{L}$. & Vinca & En \\
\hline ARACEAE & Aglaonema commutatum Schott & Dólar & $\mathrm{Hb}$ \\
\hline ARACEAE & Anthurium andraeanum Linden ex André * & Anturio & $\mathrm{Hb}$ \\
\hline ARACEAE & Anthurium crassinervium (Jacq.) Schott ${ }^{*}$ & Anturio & $\mathrm{Hb}$ \\
\hline ARACEAE & Anthurium scherzerianum Schott * & Anturio cola de marrano & $\mathrm{Hb}$ \\
\hline ARACEAE & Colocasia esculenta (L.) Schott & Bore, miami & $\mathrm{Hb}$ \\
\hline ARACEAE & Dieffenbachia oerstedii Schott * & Calatea luna & $\mathrm{Hb}$ \\
\hline ARACEAE & Dieffenbachia seguine (Jacq.) Schott * & Cucaracho, diefembaquia & $\mathrm{Hb}$ \\
\hline ARACEAE & Monstera deliciosa Liebm. * & Balazo & $\operatorname{Ln}$ \\
\hline ARACEAE & Philodendron bipinnatifidum Schott ex Endl. & Filodendro & $\mathrm{Hb}$ \\
\hline ARACEAE & Spathiphyllum wallisii Regel & Garcita, espatifilo & $\mathrm{Hb}$ \\
\hline ARACEAE & Syngonium podophyllum Schott * & Singonio & En \\
\hline ARACEAE & Zantedeschia aethiopica (L.) Spreng. & Cartucho & $\mathrm{Hb}$ \\
\hline ARACEAE & Zantedeschia elliottiana (W.Watson) Engl. & Cartucho amarillo & $\mathrm{Hb}$ \\
\hline ARACEAE & Zantedeschia rehmannii Engl. & Cartucho rosado & $\mathrm{Hb}$ \\
\hline ARALIACEAE & Fatsia japonica (Thunb.) Decne. \& Planch. & Arabia & $A b$ \\
\hline ARALIACEAE & Hedera helix L. & Hedera, hiedra & $\operatorname{Ln}$ \\
\hline ARALIACEAE & Polyscias scutellaria (Burm.f.) Fosberg & Millonaria & $\mathrm{Ab}$ \\
\hline ARALIACEAE & Schefflera actinophylla (Endl.) Harms & Cheflera & Ar \\
\hline ARALIACEAE & Schefflera arboricola (Hayata) Merr. & Cheflera árbol & $\mathrm{Ab}$ \\
\hline ARALIACEAE & $\begin{array}{l}\text { Schefflera elegantissima (Veitch ex Mast.) Lowry \& } \\
\text { Frodin }\end{array}$ & Falsa aralia & $A b$ \\
\hline ARAUCARIACEAE & Araucaria heterophylla (Salisb.) Franco & Araucaria & $\mathrm{Ar}$ \\
\hline ARECACEAE & Ceroxylon quindiuense (H.Karst.) H.Wendl. * & Palma de cera & $\mathrm{Ar}$ \\
\hline ARECACEAE & Phoenix canariensis Chabaud & Palma & $\mathrm{Ar}$ \\
\hline ARECACEAE & Phoenix roebelenii O'Brien & Palma rivelina & $\mathrm{Ab}$ \\
\hline ARECACEAE & $\begin{array}{l}\text { Washingtonia filifera (Linden ex André) H.Wendl. ex } \\
\text { de Bary }\end{array}$ & Washintonia & $\mathrm{Ar}$ \\
\hline ASPARAGACEAE & Agave americana L. & Agave, maguey, pita & Sc \\
\hline ASPARAGACEAE & Agave attenuata Salm-Dyck & Agave & Sc \\
\hline ASPARAGACEAE & Albuca bracteata (Thunb.) J.C. Manning \& Goldblatt & Planta de la prosperidad & $\mathrm{Hb}$ \\
\hline ASPARAGACEAE & Asparagus densiflorus (Kunth) Jessop & Esparraguera, aspárrago & $\mathrm{Hb}$ \\
\hline ASPARAGACEAE & Asparagus densiflorus var. sprengeri Kunth & Esparraguera, aspárrago & $\mathrm{Hb}$ \\
\hline ASPARAGACEAE & Asparagus setaceus (Kunth) Jessop & Brisa, espuma de mar & $\mathrm{Hb}$ \\
\hline ASPARAGACEAE & Beaucarnea recurvata Lem. & $\begin{array}{l}\text { Palma pony, cabeza de } \\
\text { indio }\end{array}$ & $\mathrm{Ar}$ \\
\hline ASPARAGACEAE & Chlorophytum comosum (Thunb.) Jacques & Cinta & $\mathrm{Hb}$ \\
\hline ASPARAGACEAE & Cordyline australis (G.Forst.) Endl. & Yuca & Ar \\
\hline ASPARAGACEAE & Cordyline fruticosa (L.) A.Chev. & Palma bicolor & $\mathrm{Ab}$ \\
\hline ASPARAGACEAE & Dracaena braunii Engl. & Lucky bamboo & Sf \\
\hline
\end{tabular}




\begin{tabular}{|c|c|c|c|}
\hline Familia & Nombre científico & Nombre común & Hábito** \\
\hline ASPARAGACEAE & Dracaena fragrans (L.) Ker Gawl. & $\begin{array}{l}\text { Llanecrey, árbol de la } \\
\text { felicidad }\end{array}$ & Sf \\
\hline ASPARAGACEAE & Dracaena reflexa Lam. & Canción de la India & Sf \\
\hline ASPARAGACEAE & Furcraea hexapetala (Jacq.) Urb. * & Fique & Sc \\
\hline ASPARAGACEAE & Ornithogalum thyrsoides Jacq. & Estrella de David & $\mathrm{Hb}$ \\
\hline ASPARAGACEAE & Sansevieria cylindrica Bojer ex Hook. & Lanza africana & Sc \\
\hline ASPARAGACEAE & Sansevieria trifasciata Prain & Lengua de suegra & Sc \\
\hline ASPARAGACEAE & Yucca gigantea Lem. * & Yuca, palma yuca & Ar \\
\hline ASPHODELACEAE & Aloe arborescens Mill. & Sábila & Sc \\
\hline ASPHODELACEAE & Aloe vera (L.) Burm.f. & Aloe, sábila & Sc \\
\hline ASPHODELACEAE & Gasteria acinacifolia (J.Jacq.) Haw. & Suculenta & Sc \\
\hline ASPHODELACEAE & Haworthia fasciata (Willd.) Haw. & Sábila cebra & Sc \\
\hline ASPHODELACEAE & Hemerocallis lilioasphodelus L. & Azucena amarilla & $\mathrm{Hb}$ \\
\hline ASPHODELACEAE & Kniphofia uvaria (L.) Oken & Llamas & $\mathrm{Hb}$ \\
\hline ASPHODELACEAE & Phormium tenax J.R.Forst. \& G.Forst. & Lino & $\mathrm{Hb}$ \\
\hline ASPLENIACEAE & Asplenium nidus L. & Nido de ave & $\mathrm{Hb}$ \\
\hline ASTERACEAE & Achillea millefolium $\mathrm{L}$. & Milenramas & $\mathrm{Hb}$ \\
\hline ASTERACEAE & Argyranthemum frutescens (L.) Sch. Bip. & Margarita, argirantemon & $\mathrm{Ab}$ \\
\hline ASTERACEAE & Aster amellus L. & Áster & $\mathrm{Hb}$ \\
\hline ASTERACEAE & Baccharis latifolia (Ruiz \& Pav.) Pers. * & Chilco & $\mathrm{Ab}$ \\
\hline ASTERACEAE & Calendula officinalis L. & Caléndula & $\mathrm{Hb}$ \\
\hline ASTERACEAE & Callistephus chinensis (L.) Nees & Margarita & $\mathrm{Hb}$ \\
\hline ASTERACEAE & Centaurea cineraria $\mathrm{L}$. & Cineraria marítima & $\mathrm{Hb}$ \\
\hline ASTERACEAE & Chamaemelum nobile (L.) All. & Romana & $\mathrm{Hb}$ \\
\hline ASTERACEAE & Chrysanthemum morifolium Ramat. & Crisantemo & Sf \\
\hline ASTERACEAE & Coreopsis auriculata L. & Bella Diana & $\mathrm{Hb}$ \\
\hline ASTERACEAE & Dahlia imperialis Roezl ex Ortgies * & Dalia & $\mathrm{Hb}$ \\
\hline ASTERACEAE & Dahlia pinnata Cav. & Dalia & $\mathrm{Hb}$ \\
\hline ASTERACEAE & Erigeron karvinskianus DC. * & Margarita, manzanilla & $\mathrm{Hb}$ \\
\hline ASTERACEAE & Felicia drakensbergensis J.M. Wood \& M.S. Evans & Margarita azul & Sf \\
\hline ASTERACEAE & Gaillardia x grandiflora Hort. ex Van Houtte & Gallardia & $\mathrm{Hb}$ \\
\hline ASTERACEAE & Gazania rigens (L.) Gaertn. & Gazania & $\mathrm{Hb}$ \\
\hline ASTERACEAE & Gerbera jamesonii Bolus ex Hook.f. & Gerbera & $\mathrm{Hb}$ \\
\hline ASTERACEAE & Helianthus annuus L. & Girasol & $\mathrm{Hb}$ \\
\hline ASTERACEAE & Helichrysum bracteatum (Vent.) Haw. & Inmortal, botón de oro & $\mathrm{Hb}$ \\
\hline ASTERACEAE & Helichrysum petiolare Hilliard \& B.L. Burtt & Eclipso & $A b$ \\
\hline ASTERACEAE & Ismelia carinata (Schousb.) Sch.Bip. & Margarita & $\mathrm{Hb}$ \\
\hline ASTERACEAE & Jacobaea maritima (L.) Pelser \& Meijden & Cineraria & Sf \\
\hline ASTERACEAE & Leucanthemum vulgare (Vaill.) Lam. & Margarita común & $\mathrm{Hb}$ \\
\hline ASTERACEAE & Leucophyta brownii Cass. & Helecho gris, alambrito & $\mathrm{Ab}$ \\
\hline ASTERACEAE & Osteospermum fruticosum (L.) Norl. & Margarita punto azul & Sf \\
\hline
\end{tabular}




\begin{tabular}{|c|c|c|c|}
\hline Familia & Nombre científico & Nombre común & Hábito** \\
\hline ASTERACEAE & Osteospermum jucundum (E. Phillips) Norl. & Margarita punto azul & Sf \\
\hline ASTERACEAE & Pericallis hybrida (Regel) B. Nord. & Cineraria & $\mathrm{Hb}$ \\
\hline ASTERACEAE & Santolina chamaecyparissus L. & Abrótano hembra & Sf \\
\hline ASTERACEAE & Senecio niveoaureus Cuatrec. ${ }^{*}$ & Frailejón & $\mathrm{Hb}$ \\
\hline ASTERACEAE & Senecio rowleyanus $\mathrm{H}$. Jacobsen & Rosario, camándula & Sc \\
\hline ASTERACEAE & Senecio tamoides DC. & $\begin{array}{l}\text { Lluvia de oro, estrella de } \\
\text { David }\end{array}$ & $\operatorname{Ln}$ \\
\hline ASTERACEAE & Silybum marianum (L.) Gaertn. & Cardosanto & $\mathrm{Hb}$ \\
\hline ASTERACEAE & Smallanthus pyramidalis (Triana) H. Rob. * & Arboloco & $\mathrm{Ar}$ \\
\hline ASTERACEAE & Tagetes erecta L. * & Tagetes & $\mathrm{Hb}$ \\
\hline ASTERACEAE & Tagetes lucida Cav. * & Anís & $\mathrm{Hb}$ \\
\hline ASTERACEAE & Zinnia peruviana (L.) L. * & Zinia & $\mathrm{Hb}$ \\
\hline BALSAMINACEAE & Impatiens sodenii Engl. \& Warb. & Tres estrellas & Sc \\
\hline BALSAMINACEAE & Impatiens walleriana Hook. f. & Bella helena, guinea & Sc \\
\hline BEGONIACEAE & Begonia boliviensis A.DC. * & Begonia santa cruz & $\mathrm{Hb}$ \\
\hline BEGONIACEAE & Begonia bowerae Ziesenh. * & Piel de sapo & $\mathrm{Hb}$ \\
\hline BEGONIACEAE & $\begin{array}{c}\text { Begonia cucullata var. hookeri (A.DC.) L.B.Sm. \& } \\
\text { B.G.Schub.* }\end{array}$ & Pichones & $\mathrm{Hb}$ \\
\hline BEGONIACEAE & Begonia foliosa Kunth * & Begonia & Sf \\
\hline BEGONIACEAE & Begonia maculata Raddi * & Ala de ángel & $\mathrm{Sf}$ \\
\hline BEGONIACEAE & Begonia masoniana Irmsch. ex Ziesenh. & Begonia cruz de hierro & $\mathrm{Hb}$ \\
\hline BEGONIACEAE & Begonia reniformis Dryand. & Begonia elatior & $\mathrm{Hb}$ \\
\hline BEGONIACEAE & Begonia rex Putz. & Begonia rex & $\mathrm{Hb}$ \\
\hline BEGONIACEAE & Begonia $\mathrm{x}$ tuberhybrida Voss* & Begonia tuberosa & $\mathrm{Hb}$ \\
\hline BETULACEAE & Alnus acuminata Kunth * & Aliso & $\mathrm{Ar}$ \\
\hline BIGNONIACEAE & Pandorea jasminoides (Lindl.) K. Schum. & Bignonia blanca & Ln \\
\hline BIGNONIACEAE & Pyrostegia venusta (Ker Gawl.) Miers & Tango & Ln \\
\hline BIGNONIACEAE & Tanaecium pyramidatum (Rich.) L.G. Lohmann * & - & Ln \\
\hline BIGNONIACEAE & Tecoma capensis (Thunb.) Lindl. & Tecomaria & $A b$ \\
\hline BIGNONIACEAE & Tecoma stans (L.) Juss. ex Kunth & Chirlobirlo, chicalá & $\mathrm{Ar}$ \\
\hline BORAGINACEAE & Cynoglossum amabile Stapf \& J.R. Drumm. & Miosotis de China & $\mathrm{Hb}$ \\
\hline BRASSICACEAE & Brassica oleracea L. & Col de jardín & $\mathrm{Hb}$ \\
\hline BRASSICACEAE & Lobularia maritima (L.) Desv. & Reseda & $\mathrm{Hb}$ \\
\hline BRASSICACEAE & Matthiola incana (L.) R.Br. & Alhelí & $\mathrm{Hb}$ \\
\hline BROMELIACEAE & Aechmea calyculata (E.Morren) Baker & Bromelia, quiche, guiche & $\mathrm{Hb}$ \\
\hline BROMELIACEAE & Aechmea fasciata (Lindl.) Baker & Bromelia, quiche, guiche & $\mathrm{Hb}$ \\
\hline BROMELIACEAE & Guzmania claviformis H.E. Luther* & Bromelia, quiche, guiche & $\mathrm{Hb}$ \\
\hline BROMELIACEAE & Neoregelia carolinae (Beer) L.B. Sm. ${ }^{*}$ & Bromelia, quiche, guiche & $\mathrm{Hb}$ \\
\hline BROMELIACEAE & Tillandsia cyanea Linden ex K.Koch * & Bromelia, quiche, guiche & $\mathrm{Hb}$ \\
\hline BROMELIACEAE & Tillandsia leiboldiana Schltdl. * & Bromelia, quiche, guiche & $\mathrm{Hb}$ \\
\hline BROMELIACEAE & Vriesea splendens (Brongn.) Lem. & Bromelia, quiche, guiche & $\mathrm{Hb}$ \\
\hline
\end{tabular}




\begin{tabular}{|c|c|c|c|}
\hline Familia & Nombre científico & Nombre común & Hábito** \\
\hline CACTACEAE & Acanthocereus tetragonus (L.) Hummelinck * & Cactus & Sc \\
\hline CACTACEAE & Austrocylindropuntia cylindrica (Lam.) Backeb. * & Cactus & Sc \\
\hline CACTACEAE & Austrocylindropuntia subulata (Muehlenpf.) Backeb.* & Cactus & Sc \\
\hline CACTACEAE & Browningia hernandezii Fern.Alonso * & Cactus & Sc \\
\hline CACTACEAE & Cereus hexagonus (L.) Mill. * & Cactus & Sc \\
\hline CACTACEAE & Cylindropuntia sp. & Cactus & Sc \\
\hline CACTACEAE & Epiphyllum sp. * & Pitaya & Sc \\
\hline CACTACEAE & $\begin{array}{l}\text { Gymnocalycium mihanovichii (Frič \& Gürke) Britton } \\
\text { \& Rose }\end{array}$ & Cactus & Sc \\
\hline CACTACEAE & Hatiora gaertneri (Regel) Barthlott & Cactus de navidad & Sc \\
\hline CACTACEAE & Hylocereus sp. * & Cactus & Sc \\
\hline CACTACEAE & Mammillaria mammillaris (L.) H.Karst. * & Cactus & Sc \\
\hline CACTACEAE & Melocactus sp. ${ }^{*}$ & Cactus & Sc \\
\hline CACTACEAE & Opuntia caracassana Salm-Dyck * & Cactus & Sc \\
\hline CACTACEAE & Opuntia microdasys (Lehm.) Pfeiff. & Cactus & Sc \\
\hline CACTACEAE & $\begin{array}{l}\text { Opuntia microdasys subsp. rufida (Engelm.) U. } \\
\text { Guzmán \& Mandujano }\end{array}$ & Cactus & Sc \\
\hline CACTACEAE & Opuntia pubescens H.L. Wendl. ex Pfeiff. * & Cactus & Sc \\
\hline CACTACEAE & Pilosocereus lanuginosus (L.) Byles \& G.D. Rowley & Cactus & Sc \\
\hline CACTACEAE & Schlumbergera truncata (Haw.) Moran & $\begin{array}{l}\text { Cactus de navidad, } \\
\text { filocactus }\end{array}$ & Sc \\
\hline CACTACEAE & Stenocereus griseus (Haw.) Buxb. ${ }^{*}$ & Cactus & Sc \\
\hline CACTACEAE & Trichocereus sp. * & Cactus & Sc \\
\hline CALCEOLARIACEAE & Calceolaria $\mathrm{x}$ herbeohybrida Voss. & Carrielitos, globitos & $\mathrm{Hb}$ \\
\hline CAMPANULACEAE & Campanula isophylla Moretti & $\begin{array}{l}\text { Estrella de Italia, } \\
\text { campanilla }\end{array}$ & $\mathrm{Hb}$ \\
\hline CAMPANULACEAE & Lobelia erinus L. & Zulias & $\mathrm{Hb}$ \\
\hline CANNACEAE & Canna indica L. * & Achira & $\mathrm{Hb}$ \\
\hline CAPRIFOLIACEAE & Lonicera japonica Thunb. & Madre selva & $\operatorname{Ln}$ \\
\hline CAPRIFOLIACEAE & Scabiosa atropurpurea L. & Alfiletero, cardo & $\mathrm{Hb}$ \\
\hline CARYOPHYLLACEAE & Dianthus barbatus L. & Clavel de poeta & $\mathrm{Hb}$ \\
\hline CARYOPHYLLACEAE & Dianthus caryophyllus L. & Clavel común & $\mathrm{Hb}$ \\
\hline CARYOPHYLLACEAE & Dianthus chinensis L. & Clavelina & $\mathrm{Hb}$ \\
\hline CARYOPHYLLACEAE & Dianthus gratianopolitanus Vill. & Clavelina & $\mathrm{Hb}$ \\
\hline CASUARINACEAE & Casuarina equisetifolia L. & Pino australiano & $\mathrm{Hb}$ \\
\hline CELASTRACEAE & Euonymus japonicus Thunb. & $\begin{array}{l}\text { Evónimo del japón, } \\
\text { evónimo }\end{array}$ & $A b$ \\
\hline CLUSIACEAE & Clusia orthoneura Standl. * & Clusia & $\mathrm{Ab}$ \\
\hline COMMELINACEAE & Tradescantia fluminensis Vell. & Cinta & $\mathrm{Hb}$ \\
\hline COMMELINACEAE & Tradescantia pallida (Rose) D.R. Hunt & Purpurina & $\mathrm{Hb}$ \\
\hline
\end{tabular}




\begin{tabular}{|c|c|c|c|}
\hline Familia & Nombre científico & Nombre común & Hábito** \\
\hline COMMELINACEAE & Tradescantia zebrina Bosse & $\begin{array}{l}\text { Suelda consuelda, } \\
\text { panameña }\end{array}$ & $\mathrm{Hb}$ \\
\hline CONVOLVULACEAE & Convolvulus tricolor $\mathrm{L}$. & $\begin{array}{l}\text { Campanilla, don Diego del } \\
\text { día }\end{array}$ & En \\
\hline CONVOLVULACEAE & Ipomoea purpurea (L.) Roth & Campanilla & En \\
\hline CRASSULACEAE & Aeonium arboreum Webb \& Berthel. & Suculenta & Sc \\
\hline CRASSULACEAE & Aeonium canariense (L.) Webb \& Berthel. & Chupahuevo & Sc \\
\hline CRASSULACEAE & Aeonium tabuliforme (Haw.) Webb \& Berthel. & Rosa de Jericó & Sc \\
\hline CRASSULACEAE & $\begin{array}{c}\text { Bryophyllum daigremontianum (Raym.-Hamet \& } \\
\text { H.Perrier) A. Berger }\end{array}$ & Suculenta & Sc \\
\hline CRASSULACEAE & Bryophyllum delagoense (Eckl. \& Zeyh.) Druce & Suculenta & Sc \\
\hline CRASSULACEAE & $\begin{array}{c}\text { Bryophyllum fedtschenkoi (Raym.-Hamet \& H. } \\
\text { Perrier) Lauz.-March. }\end{array}$ & Suculenta & Sc \\
\hline CRASSULACEAE & Bryophyllum pinnatum (Lam.) Oken & Suculenta & Sc \\
\hline CRASSULACEAE & Cotyledon orbiculata L. & Suculenta & Sc \\
\hline CRASSULACEAE & $\begin{array}{c}\text { Crassula capitella subsp. thyrsiflora (Thunb.) } \\
\text { Toelken }\end{array}$ & Estrella ninja & Sc \\
\hline CRASSULACEAE & Crassula multicava Lem. & Suculenta & Sc \\
\hline CRASSULACEAE & Crassula ovata (Mill.) Druce & Bastoncito, orejas de shrek & Sc \\
\hline CRASSULACEAE & Echeveria grandiflora Haw. & Suculenta & Sc \\
\hline CRASSULACEAE & Echeveria pallida E.Walther & Rosa verde & $\mathrm{Sc}$ \\
\hline CRASSULACEAE & Kalanchoe blossfeldiana Poelln. & Coralitos & Sc \\
\hline CRASSULACEAE & Kalanchoe tomentosa Baker & Orejas de conejo & Sc \\
\hline CRASSULACEAE & Sedum lineare Thunb. & Uña de señorita & Sc \\
\hline CRASSULACEAE & Sedum morganianum E.Walther & Trenzas de María & $\mathrm{Sc}$ \\
\hline CRASSULACEAE & Sedum palmeri S. Watson & Suculenta & Sc \\
\hline CRASSULACEAE & Sedum rubrotinctum R.T. Clausen & Platanito, bananito & Sc \\
\hline CUPRESSACEAE & Cupressus lusitanica Mill.* & Pino ciprés & $\mathrm{Ar}$ \\
\hline CUPRESSACEAE & Cupressus macrocarpa Hartw. & Cedro limón & $\operatorname{Ar}$ \\
\hline CUPRESSACEAE & Cupressus sempervirens L. & Pino vela & $\mathrm{Ar}$ \\
\hline CUPRESSACEAE & Juniperus squamata Buch.-Ham. ex D.Don & Pino san José & $\mathrm{Ab}$ \\
\hline CUPRESSACEAE & Platycladus orientalis (L.) Franco & Pino libro & $A b$ \\
\hline CYCADACEAE & Cycas rumphii Miq. & $\begin{array}{l}\text { Palma cica, espina de } \\
\text { pescado }\end{array}$ & $\mathrm{Hb}$ \\
\hline CYPERACEAE & Cyperus involucratus Rottb. & Papiro chino & $\mathrm{Hb}$ \\
\hline CYPERACEAE & Cyperus papyrus L. & $\begin{array}{l}\text { Papiro, papiro de los } \\
\text { egipcios }\end{array}$ & $\mathrm{Hb}$ \\
\hline DAVALLIACEAE & Davallia canariensis (L.) Sm. & Cola de mono & $\mathrm{Hb}$ \\
\hline DROSERACEAE & Dionaea muscipula J. Ellis & Venus & $\mathrm{Hb}$ \\
\hline DROSERACEAE & Drosera capensis L. & Atrapamoscas & $\mathrm{Hb}$ \\
\hline DROSERACEAE & Drosera glabripes (Harv. ex Planch.) Stein & Atrapamoscas & $\mathrm{Hb}$ \\
\hline
\end{tabular}




\begin{tabular}{|c|c|c|c|}
\hline Familia & Nombre científico & Nombre común & Hábito** \\
\hline ELAEOCARPACEAE & Vallea stipularis L.f. * & $\begin{array}{l}\text { Campano, sanjuanito, } \\
\text { raque }\end{array}$ & Ar \\
\hline ERICACEAE & Rhododendron simsii Planch. & Azalea & $\mathrm{Ab}$ \\
\hline ESCALLONIACEAE & Escallonia pendula (Ruiz \& Pav.) Pers. * & Mangle & $\operatorname{Ar}$ \\
\hline ESCALLONIACEAE & Escallonia polifolia Hook. & Tibar & $A b$ \\
\hline EUPHORBIACEAE & Acalypha chamaedrifolia (Lam.) Müll. Arg. & Churrusquitos, gusanitos & Sf \\
\hline EUPHORBIACEAE & Acalypha wilkesiana Müll.Arg. & Millón tailandés & $\mathrm{Ab}$ \\
\hline EUPHORBIACEAE & Codiaeum variegatum (L.) Rumph. ex A. Juss. & Croto variegado, colorama & $\mathrm{Ab}$ \\
\hline EUPHORBIACEAE & Croton coriaceus Kunth * & Sangregrado, drago, croto & Ar \\
\hline EUPHORBIACEAE & Euphorbia cotinifolia L. * & Lechero rojo & $A b$ \\
\hline EUPHORBIACEAE & Euphorbia milii Des Moul. & Corona de espinas & $\mathrm{Ab}$ \\
\hline EUPHORBIACEAE & Euphorbia pulcherrima Willd. ex Klotzsch & $\begin{array}{l}\text { Navidad, sombrilla } \\
\text { japonesa }\end{array}$ & $A b$ \\
\hline EUPHORBIACEAE & Euphorbia tirucalli L. & Árbol desnudo & Sc \\
\hline EUPHORBIACEAE & Euphorbia tithymaloides L. & Bítamo & Sc \\
\hline EUPHORBIACEAE & Euphorbia trigona Mill. & Cactus & Sc \\
\hline EUPHORBIACEAE & Ricinus communis L. & Ricino, higuerillo & $\mathrm{Ab}$ \\
\hline FABACEAE & Acacia baileyana F. Muell. & Acacia morada & Ar \\
\hline FABACEAE & Acacia longifolia (Andrews) Willd. & Acacia & $\mathrm{Ar}$ \\
\hline FABACEAE & Acacia mearnsii De Wild. & Acacia & $\mathrm{Ar}$ \\
\hline FABACEAE & Acacia melanoxylon R. Br. & Acacia japonesa & $\mathrm{Ar}$ \\
\hline FABACEAE & Caesalpinia spinosa (Molina) Kuntze * & Dividivi & $\mathrm{Ar}$ \\
\hline FABACEAE & Erythrina rubrinervia Kunth * & Chocho & Ar \\
\hline FABACEAE & Inga sp. & Guamo & $\mathrm{Ar}$ \\
\hline FABACEAE & Lotus berthelotii Masf. & Manto de gitana & $\mathrm{Hb}$ \\
\hline FABACEAE & Paraserianthes lophantha (Willd.) I.C.Nielsen & Acacia bracatinga & $\mathrm{Ar}$ \\
\hline FABACEAE & Senna multiglandulosa (Jacq.) H.S. Irwin \& Barneby * & Alcaparro enano & $\mathrm{Ar}$ \\
\hline FABACEAE & Senna viarum (Little) H.S. Irwin \& Barneby * & Alcaparro gigante & Ar \\
\hline FAGACEAE & Quercus humboldtii Bonpl. * & Roble & $\mathrm{Ar}$ \\
\hline GERANIACEAE & Pelargonium x domesticum L.H. Bailey & Geranio & $\mathrm{Hb}$ \\
\hline GERANIACEAE & Pelargonium odoratissimum (L.) L’Hér. & Aroma & $\mathrm{Hb}$ \\
\hline GERANIACEAE & Pelargonium peltatum (L.) L’Hér. & Hiedra & $\mathrm{Hb}$ \\
\hline GERANIACEAE & Pelargonium zonale (L.) L'Hér. ex Aiton & Novios & $\mathrm{Hb}$ \\
\hline GESNERIACEAE & $\begin{array}{l}\text { Kohleria amabilis var. bogotensis (G. Nicholson) L.P. } \\
\text { Kvist \& L.E. Skog * }\end{array}$ & Coleria & $\mathrm{Hb}$ \\
\hline GESNERIACEAE & Nematanthus gregarius D.L. Denham & Pescaditos & $\mathrm{Hb}$ \\
\hline GESNERIACEAE & Saintpaulia ionantha $\mathrm{H}$. Wendl. & Violeta africana & $\mathrm{Hb}$ \\
\hline GESNERIACEAE & Sinningia speciosa (Lodd.) Hiern & Gloxinia & $\mathrm{Hb}$ \\
\hline HAEMODORACEAE & Anigozanthos flavidus DC. & Anizogantos & $\mathrm{Hb}$ \\
\hline HYDRANGEACEAE & Hydrangea macrophylla (Thunb.) Ser. & Hortensia & $\mathrm{Hb}$ \\
\hline
\end{tabular}




\begin{tabular}{|c|c|c|c|}
\hline Familia & Nombre científico & Nombre común & Hábito** \\
\hline HYPERICACEAE & Hypericum patulum Thunb. & Hipérico & $A b$ \\
\hline IRIDACEAE & Crocosmia $\times$ crocosmiiflora (Lemoine) N.E. Br. & Chispita & $\mathrm{Hb}$ \\
\hline IRIDACEAE & Dietes bicolor (Steud.) Sweet ex Klatt & Iris africano & $\mathrm{Hb}$ \\
\hline IRIDACEAE & Freesia refracta (Jacq.) Klatt & Nardo & $\mathrm{Hb}$ \\
\hline IRIDACEAE & Gladiolus x hybridus C. Morren & Gladiolo & $\mathrm{Hb}$ \\
\hline IRIDACEAE & Iris $\mathrm{x}$ germanica $\mathrm{L}$. & Lirio barbudo & $\mathrm{Hb}$ \\
\hline IRIDACEAE & Iris japonica Thunb. & Flor de mariposa & $\mathrm{Hb}$ \\
\hline JUGLANDACEAE & Juglans neotropica Diels* & Nogal, cedro nogal & $\operatorname{Ar}$ \\
\hline LAMIACEAE & Clerodendrum thomsoniae Balf.f. & Corazón herido & En \\
\hline LAMIACEAE & Lamium galeobdolon (L.) L. & Aluminio & En \\
\hline LAMIACEAE & Plectranthus madagascariensis (Pers.) Benth. & Incienso & $\mathrm{Hb}$ \\
\hline LAMIACEAE & Plectranthus parviflorus Willd. & Planta del dinero & $\mathrm{Hb}$ \\
\hline LAMIACEAE & Plectranthus scutellarioides (L.) R. Br. & Pompaduro & $\mathrm{Hb}$ \\
\hline LAMIACEAE & Rosmarinus officinalis L. & Romero & $\mathrm{Ab}$ \\
\hline LAMIACEAE & Salvia leucantha Cav. & Salvia & $\mathrm{Hb}$ \\
\hline LAMIACEAE & Salvia splendens Sellow ex Schult. & Liberales, salvia & $\mathrm{Hb}$ \\
\hline LAMIACEAE & Stachys byzantina K. Koch & Hierbas de la perlesía & $\mathrm{Hb}$ \\
\hline LAMIACEAE & Westringia fruticosa (Willd.) Druce & Romero australiano & $\mathrm{Ab}$ \\
\hline LILIACEAE & Lilium candidum L. & Lirio & $\mathrm{Hb}$ \\
\hline LYTHRACEAE & Cuphea ignea A. DC. * & Cigarrillitos & $\mathrm{Hb}$ \\
\hline LYTHRACEAE & Lafoensia acuminata (Ruiz \& Pav.) DC. * & Guayacán de Manizales & $\mathrm{Ar}$ \\
\hline LYTHRACEAE & Punica granatum L. & Granado & $A b$ \\
\hline MAGNOLIACEAE & Liriodendron tulipifera $\mathrm{L}$. & Tulipero & $\mathrm{Ar}$ \\
\hline MAGNOLIACEAE & Magnolia grandiflora L. & Magnolia & $\mathrm{Ar}$ \\
\hline MALVACEAE & Abutilon hybridum Voss & Abutilón & $\mathrm{Ab}$ \\
\hline MALVACEAE & $\begin{array}{l}\text { Abutilon megapotamicum (A.Spreng.) A.St.-Hil. \& } \\
\text { Naudin }\end{array}$ & Farolitos & $A b$ \\
\hline MALVACEAE & Abutilon pictum (Gillies ex Hook.) Walp. * & Abutilón variegado & $\mathrm{Ab}$ \\
\hline MALVACEAE & Alcea rosea $\mathrm{L}$. & Malva & $\mathrm{Hb}$ \\
\hline MALVACEAE & Anisodontea capensis (L.) D.M. Bates & Malva imperial, malvastro & $\mathrm{Ab}$ \\
\hline MALVACEAE & Grewia glandulosa Vahl & - & $\mathrm{Ab}$ \\
\hline MALVACEAE & Hibiscus rosa-sinensis $\mathrm{L}$. & Cayeno & $\mathrm{Ab}$ \\
\hline MALVACEAE & Malvaviscus penduliflorus Moc. \& Sessé ex DC. & Malvavisco & $\mathrm{Ab}$ \\
\hline MARANTACEAE & Calathea zebrina (Sims) Lindl. & Calatea pana, cola de lapa & $\mathrm{Hb}$ \\
\hline MARANTACEAE & Ctenanthe setosa (Roscoe) Eichler & Diez mandamientos & $\mathrm{Hb}$ \\
\hline MARANTACEAE & $\begin{array}{c}\text { Goeppertia makoyana (E. Morren) Borchs. \& S. } \\
\text { Suárez }\end{array}$ & Calatea papelito & $\mathrm{Hb}$ \\
\hline MARANTACEAE & Goeppertia roseopicta (Linden) Borchs. \& S. Suárez & Medallón & $\mathrm{Hb}$ \\
\hline MARANTACEAE & Maranta leuconeura E. Morren & Soldaditos & $\mathrm{Hb}$ \\
\hline MELASTOMATACEAE & Centradenia grandifolia (Schltdl.) Endl. * & Siete cueros rastrero & $\mathrm{Hb}$ \\
\hline MELASTOMATACEAE & Meriania nobilis Triana * & Amarrabollo & $\mathrm{Ab}$ \\
\hline
\end{tabular}




\begin{tabular}{|c|c|c|c|}
\hline Familia & Nombre científico & Nombre común & Hábito** \\
\hline MELASTOMATACEAE & Tibouchina lepidota (Bonpl.) Baill. * & Siete cueros & $A b$ \\
\hline MELIACEAE & Cedrela montana Moritz ex Turcz. * & Cedro & $\mathrm{Ar}$ \\
\hline MORACEAE & Ficus benjamina $\mathrm{L}$. & Ficus variegado, ficus & Ar \\
\hline MORACEAE & Ficus elastica Roxb. ex Hornem. & Ficus de caucho, caucho & $\mathrm{Ar}$ \\
\hline MORACEAE & Ficus pumila $\mathrm{L}$. & $\begin{array}{l}\text { Ficus trepador, ficus } \\
\text { rastrero }\end{array}$ & $\operatorname{Ln}$ \\
\hline MORACEAE & Ficus soatensis Dugand* & Caucho & Ar \\
\hline MORACEAE & Ficus tequendamae Dugand * & Caucho & $\mathrm{Ar}$ \\
\hline MORACEAE & Ficus velutina Humb. \& Bonpl. ex Willd. * & Caucho & Ar \\
\hline MYRTACEAE & Callistemon citrinus (Curtis) Skeels & Limpiatubos & $\mathrm{Ab}$ \\
\hline MYRTACEAE & Callistemon viminalis (Sol. ex Gaertn.) G. Don & Limpiatubos, árbol llorón & $\mathrm{Ab}$ \\
\hline MYRTACEAE & $\begin{array}{c}\text { Corymbia ficifolia (F. Muell.) K.D. Hill \& L.A.S. } \\
\text { Johnson }\end{array}$ & Eucalipto & $\mathrm{Ar}$ \\
\hline MYRTACEAE & Eucalyptus cinerea F. Muell. ex Benth. & Eucalipta hembra & $\mathrm{Ar}$ \\
\hline MYRTACEAE & Eucalyptus globulus Labill. & Eucalipto & Ar \\
\hline MYRTACEAE & Leptospermum scoparium J.R. Forst. \& G. Forst. & Electrospermo, rosa china & $\mathrm{Ab}$ \\
\hline MYRTACEAE & Syzygium paniculatum Gaertn. & Eugenia & $\mathrm{Ab}$ \\
\hline NEPENTHACEAE & Nepenthes sp. & Nepentes & $\mathrm{Hb}$ \\
\hline NEPHROLEPIDACEAE & Nephrolepis cordifolia (L.) C. Presl & Helecho peine & $\mathrm{Hb}$ \\
\hline NEPHROLEPIDACEAE & Nephrolepis exaltata (L.) Schott & Helecho Boston & $\mathrm{Hb}$ \\
\hline NEPHROLEPIDACEAE & Nephrolepis exaltata var. bostoniensis Davenp. & Helecho Boston & $\mathrm{Hb}$ \\
\hline NYCTAGINACEAE & Bougainvillea glabra Choisy & Buganvil & $\operatorname{Ln}$ \\
\hline NYCTAGINACEAE & Mirabilis jalapa L. * & Don Diego de noche & $\mathrm{Ab}$ \\
\hline OLEACEAE & Fraxinus chinensis Roxb. & Urapán, fresno & $\mathrm{Ar}$ \\
\hline OLEACEAE & Jasminum polyanthum Franch. & Jazmín & $\operatorname{Ln}$ \\
\hline OLEACEAE & Ligustrum japonicum Thunb. & Aligustre & $\mathrm{Ab}$ \\
\hline OLEACEAE & Ligustrum lucidum W.T. Aiton & $\begin{array}{l}\text { Ligustro, manzano de } \\
\text { jardín }\end{array}$ & $A b$ \\
\hline OLEACEAE & Olea europaea L. & Olivo & $\mathrm{Ab}$ \\
\hline ONAGRACEAE & Fuchsia corymbiflora Ruiz \& Pav. & Zarcillejo, fucsia arbustiva & $\mathrm{Ab}$ \\
\hline ONAGRACEAE & Fuchsia magellanica Lam. & Fucsia, zarcillo & $\mathrm{Ab}$ \\
\hline ONAGRACEAE & Fuchsia microphylla Kunth & Zarcillo mini & $\mathrm{Ab}$ \\
\hline ONAGRACEAE & Fuchsia triphylla L. * & Fucsia, zarcillo & $\mathrm{Ab}$ \\
\hline ONAGRACEAE & Fuchsia $\mathrm{x}$ hybrida Voss & Fucsia, zarcillo & $\mathrm{Ab}$ \\
\hline ORCHIDACEAE & Acineta superba (Kunth) Rchb. f. ${ }^{*}$ & Toritos & $\mathrm{Hb}$ \\
\hline ORCHIDACEAE & Anguloa clowesii Lindl. * & $\begin{array}{l}\text { Cuna de venus, orquídea } \\
\text { tulipán }\end{array}$ & $\mathrm{Hb}$ \\
\hline ORCHIDACEAE & Arundina graminifolia (D. Don) Hochr. & Orquídea bambú, arundina & $\mathrm{Hb}$ \\
\hline ORCHIDACEAE & Cattleya trianae Linden \& Rchb. f. * & Flor de mayo & $\mathrm{Hb}$ \\
\hline ORCHIDACEAE & Cattleya x hybrida H.J. Veitch * & Flor de mayo & $\mathrm{Hb}$ \\
\hline ORCHIDACEAE & Epidendrum sp. * & Orquídea & $\mathrm{Hb}$ \\
\hline
\end{tabular}




\begin{tabular}{|c|c|c|c|}
\hline Familia & Nombre científico & Nombre común & Hábito** \\
\hline ORCHIDACEAE & Masdevallia sp. ${ }^{*}$ & Masdevalia & $\mathrm{Hb}$ \\
\hline ORCHIDACEAE & Maxillaria sp. * & Orquídea & $\mathrm{Hb}$ \\
\hline ORCHIDACEAE & Miltoniopsis vexillaria (Rchb. f.) God.-Leb. * & Josefina & $\mathrm{Hb}$ \\
\hline ORCHIDACEAE & $\begin{array}{l}\text { Oncidium alexandrae (Bateman) M.W. Chase \& N.H. } \\
\text { Williams * }\end{array}$ & Orquídea & $\mathrm{Hb}$ \\
\hline ORCHIDACEAE & Oncidium sp. * & Lluvia de estrellas & $\mathrm{Hb}$ \\
\hline ORCHIDACEAE & Paphiopedilum sp. & Zapatico de Obispo & $\mathrm{Hb}$ \\
\hline ORCHIDACEAE & Phalaenopsis sp. & Falenopsis & $\mathrm{Hb}$ \\
\hline ORCHIDACEAE & Phragmipedium sp. ${ }^{*}$ & Zapaticos & $\mathrm{Hb}$ \\
\hline ORCHIDACEAE & Trichocentrum sp. & Orquídea & $\mathrm{Hb}$ \\
\hline OXALIDACEAE & Biophytum dendroides (Kunth) DC. & - & $\mathrm{Hb}$ \\
\hline OXALIDACEAE & Oxalis articulata Savigny & Trébol & $\mathrm{Hb}$ \\
\hline OXALIDACEAE & Oxalis corniculata $\mathrm{L}$. & Trébol & $\mathrm{Hb}$ \\
\hline OXALIDACEAE & Oxalis purpurea $\mathrm{L}$. & Trébol holandés & $\mathrm{Hb}$ \\
\hline OXALIDACEAE & Oxalis spiralis Ruiz \& Pav. ex G. Don & Trébol morado & Sc \\
\hline OXALIDACEAE & Oxalis triangularis A. St.-Hil. & Trébol ecuatoriano & $\mathrm{Hb}$ \\
\hline PAPAVERACEAE & Eschscholzia californica Cham. & Amapola dorada & $\mathrm{Hb}$ \\
\hline PAPAVERACEAE & Papaver somniferum L. & Amapola & $\mathrm{Hb}$ \\
\hline PHYTOLACCACEAE & Ledenbergia seguierioides Klotzsch ex Moq. * & Mil flores & $\mathrm{Ab}$ \\
\hline PINACEAE & Pinus patula Schiede ex Schltdl. \& Cham. & Pino & $\mathrm{Ar}$ \\
\hline PINACEAE & Pinus radiata D. Don & Pino & $\mathrm{Ar}$ \\
\hline PIPERACEAE & Peperomia argyreia (Miq.) E. Morren * & Concha & $\mathrm{Hb}$ \\
\hline PIPERACEAE & Peperomia caperata Yunck. & Arrugitas & $\mathrm{Hb}$ \\
\hline PIPERACEAE & Peperomia obtusifolia (L.) A. Dietr. & Espejuelos, cucharitas & $\mathrm{Hb}$ \\
\hline PIPERACEAE & Peperomia rotundifolia (L.) Kunth & Centavitos & $\mathrm{Hb}$ \\
\hline PITTOSPORACEAE & Pittosporum undulatum Vent. & Jazmín & $\mathrm{Ar}$ \\
\hline PLANTAGINACEAE & Antirrhinum majus L. & Dragones, perritos & $\mathrm{Hb}$ \\
\hline PLANTAGINACEAE & Cymbalaria muralis G. Gaertn., B. Mey. \& Scherb. & Palomilla del muro & $\mathrm{Hb}$ \\
\hline PLANTAGINACEAE & Hebe sp. & Hebe & $\mathrm{Hb}$ \\
\hline PLANTAGINACEAE & Penstemon sp. & Dedalera & $\mathrm{Hb}$ \\
\hline PLUMBAGINACEAE & Armeria maritima (Mill.) Willd. & Globito & $\mathrm{Hb}$ \\
\hline PLUMBAGINACEAE & Limonium perezii (Stapf) F.T. Hubb. & Siempreviva, limonión & $\mathrm{Hb}$ \\
\hline PLUMBAGINACEAE & Plumbago auriculata Lam. & Jazmín azul & $A b$ \\
\hline POACEAE & Anthephora hermaphrodita (L.) Kuntze & Penisetum & $\mathrm{Hb}$ \\
\hline POACEAE & Arundo donax L. & Cañabrava & $\mathrm{Hb}$ \\
\hline POACEAE & Cenchrus setaceus (Forssk.) Morrone & Penisetum & $\mathrm{Hb}$ \\
\hline POACEAE & $\begin{array}{l}\text { Cortaderia selloana (Schult. \& Schult. f.) Asch. \& } \\
\text { Graebn. }\end{array}$ & Plumón silvestre & $\mathrm{Hb}$ \\
\hline POACEAE & Phyllostachys aurea Rivière \& C. Rivière & $\begin{array}{l}\text { Bambú ornamental, } \\
\text { chusque }\end{array}$ & $A b$ \\
\hline PODOCARPACEAE & Retrophyllum rospigliosii (Pilg.) C.N. Page * & Pino colombiano & $\mathrm{Ar}$ \\
\hline
\end{tabular}




\begin{tabular}{|c|c|c|c|}
\hline Familia & Nombre científico & Nombre común & Hábito** \\
\hline POLYGALACEAE & Polygala myrtifolia L. & Polígala & $\mathrm{Ab}$ \\
\hline POLYGONACEAE & Persicaria capitata (Buch.-Ham. ex D.Don) H.Gross & Nudosilla & $\mathrm{Hb}$ \\
\hline POLYPODIACEAE & Microsorum punctatum (L.) Copel. & Helecho lechuga & $\mathrm{Hb}$ \\
\hline POLYPODIACEAE & Phlebodium aureum (L.) J. Sm. * & Helecho blue star & $\mathrm{Hb}$ \\
\hline POLYPODIACEAE & Platycerium bifurcatum (Cav.) C.Chr. & Cacho de venado & $\mathrm{Hb}$ \\
\hline POLYPODIACEAE & Schellolepis subauriculata (Blume) J. Sm. & Helecho cortina & $\mathrm{Hb}$ \\
\hline PRIMULACEAE & Anagallis arvensis $\mathrm{L}$. & Anagallo & $\mathrm{Hb}$ \\
\hline PRIMULACEAE & Cyclamen persicum Mill. & Violeta de los Alpes & $\mathrm{Hb}$ \\
\hline PRIMULACEAE & Primula obconica Hance & Primula & $\mathrm{Hb}$ \\
\hline PROTEACEAE & Grevillea robusta A. Cunn. ex R. Br. & $\begin{array}{l}\text { Roble australiano, rabo de } \\
\text { pavo }\end{array}$ & $\mathrm{Ar}$ \\
\hline PTERIDACEAE & Adiantum raddianum C. Presl & Cilantrillo, culantrillo & $\mathrm{Hb}$ \\
\hline ROSACEAE & Cotoneaster pannosus Franch. & Joli rojo & $A b$ \\
\hline ROSACEAE & Photinia $\times$ fraseri Dress & Fotinia & $A b$ \\
\hline ROSACEAE & Prunus serotina Ehrh. & Cereza & $\mathrm{Ar}$ \\
\hline ROSACEAE & Pyracantha angustifolia (Franch.) C.K. Schneid. & $\begin{array}{c}\text { Piracanta, joli naranja, } \\
\text { mortiño }\end{array}$ & $A b$ \\
\hline ROSACEAE & Rosa sp. & Rosa & Sf \\
\hline RUBIACEAE & Coffea arabica L. & Café & $\mathrm{Ab}$ \\
\hline RUBIACEAE & Gardenia jasminoides J.Ellis & Gardenia & $A b$ \\
\hline RUTACEAE & Coleonema album (Thunb.) Bartl. \& H.L. Wendl. & Diosme & $\mathrm{Ab}$ \\
\hline RUTACEAE & Citrus $x$ limon (L.) Osbeck & Limón & $\mathrm{Ar}$ \\
\hline RUTACEAE & Citrus reticulata Blanco & Mandarina & $\mathrm{Ar}$ \\
\hline RUTACEAE & Citrus $\mathrm{x}$ aurantium $\mathrm{L}$. & Naranja & $\mathrm{Ar}$ \\
\hline SALICACEAE & Abatia parviflora Ruiz \& Pav. * & Duraznillo, velitas & $\mathrm{Ab}$ \\
\hline SALICACEAE & Populus nigra $\mathrm{L}$. & Álamo & $\mathrm{Ar}$ \\
\hline SALICACEAE & Salix humboldtiana Willd. * & Sauce & $\mathrm{Ar}$ \\
\hline SALICACEAE & Salix viminalis $\mathrm{L}$. & Mimbre & $A b$ \\
\hline SALICACEAE & Xylosma spiculifera (Tul.) Triana \& Planch. * & Corono & $A b$ \\
\hline SAPINDACEAE & Dodonaea viscosa (L.) Jacq. & Hayuelo & $\mathrm{Ab}$ \\
\hline SAXIFRAGACEAE & Bergenia crassifolia (L.) Fritsch & Bergenia & $\mathrm{Hb}$ \\
\hline SAXIFRAGACEAE & Heuchera sanguinea Engelm. & Flor de coral & $\mathrm{Hb}$ \\
\hline SAXIFRAGACEAE & Tolmiea menziesii (Pursh) Torr. \& A. Gray & Mil hojas & $\mathrm{Hb}$ \\
\hline SCROPHULLARIACEAE & Buddleja davidii Franch. & Arbustos de las mariposas & $\mathrm{Ab}$ \\
\hline SCROPHULLARIACEAE & Chaenostoma cordatum (Thunb.) Benth. & Estrella de san José & $\mathrm{Hb}$ \\
\hline SCROPHULLARIACEAE & Verbascum virgatum Stokes & Vara de san José & $\mathrm{Hb}$ \\
\hline SELAGINELLACEAE & Selaginella martensii Spring & Helecho cola de pavo & $\mathrm{Hb}$ \\
\hline SOLANACEAE & Brugmansia arborea (L.) Steud.* & Borrachero & $A b$ \\
\hline SOLANACEAE & Brugmansia sanguinea (Ruiz \& Pav.) D.Don * & Borrachero rojo & $\operatorname{Ar}$ \\
\hline SOLANACEAE & Brunfelsia pauciflora (Cham. \& Schltdl.) Benth. * & Jazmín lavanda & $A b$ \\
\hline SOLANACEAE & Calibrachoa sp. * & Calibranchoa & $\mathrm{Hb}$ \\
\hline
\end{tabular}




\begin{tabular}{|c|c|c|c|}
\hline Familia & Nombre científico & Nombre común & Hábito** \\
\hline SOLANACEAE & Capsicum annuum L. * & Ají ornamental & $\mathrm{Hb}$ \\
\hline SOLANACEAE & Cestrum nocturnum L. & Galán de la noche & $A b$ \\
\hline SOLANACEAE & lochroma gesnerioides (Kunth) Miers * & Sarcillejo & $A b$ \\
\hline SOLANACEAE & Lycianthes lycioides (L.) Hassl. * & Gurrubo & $\mathrm{Ab}$ \\
\hline SOLANACEAE & Nicandra physalodes (L.) Gaertn. * & Uchuva de perro & $\mathrm{Hb}$ \\
\hline SOLANACEAE & Nicotiana tabacum L. ${ }^{*}$ & Tabaco & $\mathrm{Hb}$ \\
\hline SOLANACEAE & Petunia hybrida Vilm. & Petunia & $\mathrm{Hb}$ \\
\hline SOLANACEAE & Solandra maxima (Moc. \& Sessé ex Dunal) P.S.Green* & Copa de oro & $\operatorname{Ln}$ \\
\hline SOLANACEAE & Solanum pseudocapsicum L. & Mirto & $A b$ \\
\hline SOLANACEAE & Solanum laxum Spreng. * & Manto de maría & $\operatorname{Ln}$ \\
\hline SOLANACEAE & Streptosolen jamesonii (Benth.) Miers * & Mermelada & $A b$ \\
\hline THEACEAE & Camellia japonica L. & Camelia, rosa camelia & $\mathrm{Ab}$ \\
\hline TROPAEOLACEAE & Tropaeolum majus L. * & Nabo, capuchinas & En \\
\hline URTICACEAE & Pilea cadierei Gagnep. \& Guillaumin & Planta de aluminio & $A b$ \\
\hline URTICACEAE & Pilea involucrata (Sims) C.H.Wright \& Dewar * & Pilea & $\mathrm{Hb}$ \\
\hline URTICACEAE & Pilea microphylla (L.) Liebm. * & Paletaria & $\mathrm{Hb}$ \\
\hline URTICACEAE & Soleirolia soleirolii (Req.) Dandy & Lágrimas de bebé & $\mathrm{Hb}$ \\
\hline VERBENACEAE & Duranta erecta L. * & Duranta & $\mathrm{Ab}$ \\
\hline VERBENACEAE & Lantana camara L. * & Sanguinaria & $A b$ \\
\hline VERBENACEAE & $\begin{array}{l}\text { Glandularia x hybrida (Groenl. \& Rümpler) G.L. } \\
\text { Nesom \& Pruski }{ }^{*}\end{array}$ & Verbena & $\mathrm{Hb}$ \\
\hline VIOLACEAE & Viola tricolor $\mathrm{L}$. & Pensamientos & $\mathrm{Hb}$ \\
\hline
\end{tabular}

